\title{
Improvement of a Touch Panel Switch with the Function of Bending and Addition
}

\author{
Hiroshi Kawano ${ }^{\mathrm{a}, *}$, Seiichi Serikawa ${ }^{\mathrm{b}}$ \\ ${ }^{a}$ MTI Corporation, Address, 2-1-15 Edamitsu, Yahata-higashi, Kitakyushu, Fukuoka, 805-8503, Japan

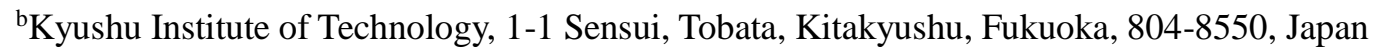 \\ *Corresponding Author: kawano@ mticorp.co.jp
}

\begin{abstract}
Recently, an advanced touch panel was proposed. It has a simple mechanism and is very flexible. The panel number can be increased or decreased arbitrarily, and the shape can be arbitrarily changed such as curved surface as well as plane. It can be used for a wearable input device by being pasted to a body. An entire surface of any shape can be used for an input device. However, it is sensitive to noise because of the simple structure. For example, it is affected by skin effect, the change in capacitance, and environmental noise. For the reason, a lot of panels cannot be connected. The improvement method has been proposed. In the model, the precision is better than that of conventional model. However, this is only simulation result. For practical use, it has the following problems. (1) Use of many number of electrical parts, and many number of connection lines. (2) Non-confirmation of the validity of the method in a real circuit. In order to improve them, a new model is proposed in this study. As the results, the followings are obtained. (1) Decrement of the number of electrical parts and the number of connection lines. The proposed new circuit system is simple. (2) Confirmation of the validity of proposed method in a real circuit. The maximum panel number is about 300. This is much more than that of conventional method.

Keywords: Touch panel, Bending, Addition
\end{abstract}

\section{Introduction}

Recently, various methods have been employed as the input interface of electronic device, for example, key-switch, voice input device, motion device, and etc. ${ }^{(1)(2)(3)}$. Touch screen is also one of them. The sensor detects the coordinates on the touched screen. Touch screen of a resistive overlay type is adopted as an input interface of the portable game machines recently. A capacitive touch screen is used for input device of smart phone. Now, it becomes popular. However, the shape is only plate. It cannot be changed to arbitrary shape. In addition, the number of panel cannot be increase easily. For the improvement, an advanced touch panel was proposed ${ }^{(4)}$. It has a simple mechanism and is very flexible. The panel number can be increased or decreased arbitrarily, and the shape can be arbitrarily changed such as curved surface as well as plane. It can be used for a wearable input device by being pasted to a body. An entire surface of any shape can be used for as an input device. However, it is sensitive to noise because of the simple structure. For example, it is affected by skin effect, the change in capacitance, and environmental noise. For the reason, a lot of panels cannot be connected. The improvement method has been proposed ${ }^{(5)}$. In the model, the precision is better than that of conventional model. However, this is only simulation result. For practical use, it has the following problems. (1) Use of many number of electrical parts, and many number of connection lines. (2) Non-confirmation of the validity of the method in a real circuit.

In order to improve them, a new model is proposed in this study. As the results, the followings are obtained. (1) Decrement of the number of electrical parts and the number of connection lines. The proposed new circuit system is simple. 
(2) Confirmation of the validity of proposed method in a real circuit. The maximum panel number is about 300. This is much more than that of conventional method. Thus, the flexible type touch panel switch can be used in practice.

\section{Proposed method}

Figure 1 shows the block diagram and parts of circuit of proposed touch panel.

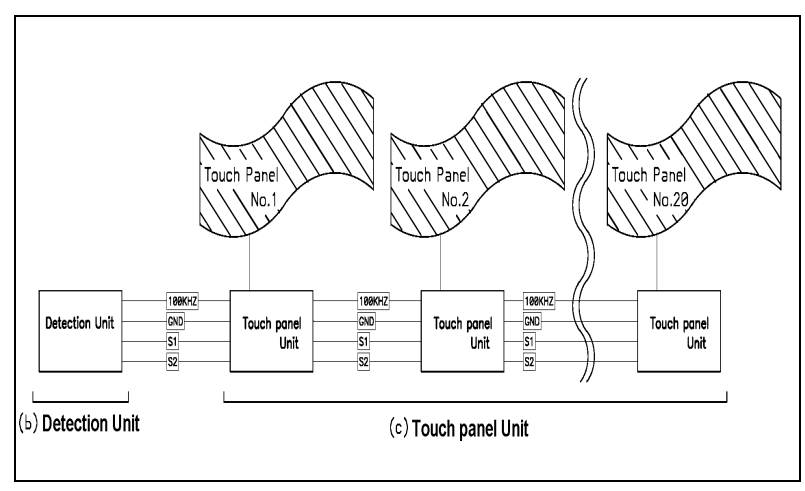

(a) Block diagram

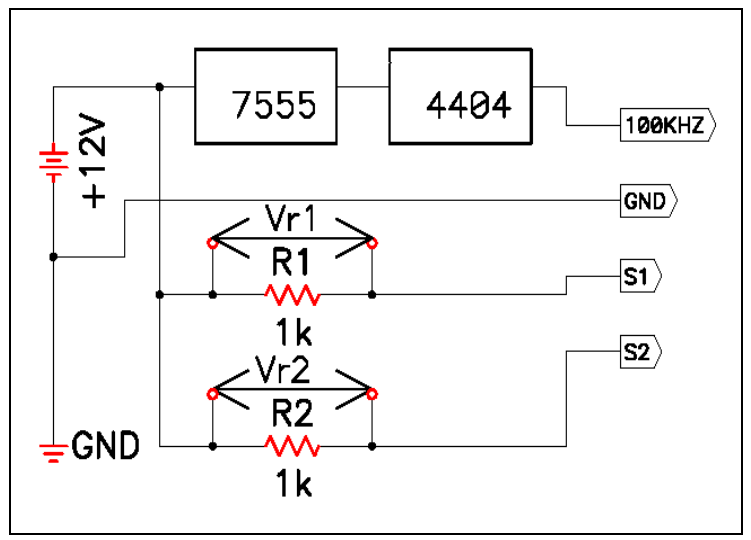

(b) Detection part

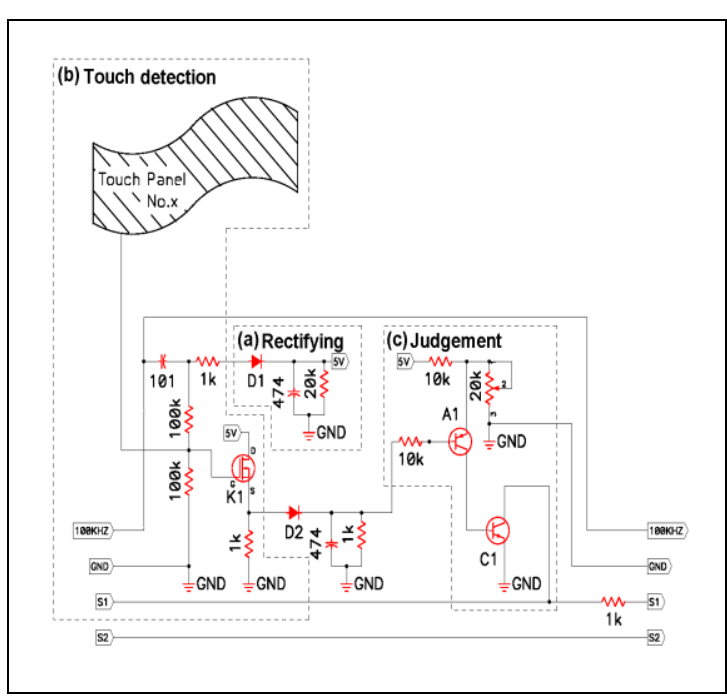

(c) Touch panel part

Figure 1 Block diagram and parts of circuit of proposed touch panel.
Table 1 Comparison of characteristics of proposed method with conventional method.

\begin{tabular}{|l|c|c|}
\hline & $\begin{array}{l}\text { The number of } \\
\text { connection } \\
\text { lines }\end{array}$ & $\begin{array}{l}\text { number of } \\
\text { electronic parts of } \\
\text { the panel part }\end{array}$ \\
\hline $\begin{array}{l}\text { conventional } \\
\text { method }\end{array}$ & 5 & more than 42 \\
\hline $\begin{array}{l}\text { proposed } \\
\text { method }\end{array}$ & 4 & 17 \\
\hline
\end{tabular}

Fig.1 (a) is the block diagram of entire device. It can be divided into two parts. One is detection part, and another is touch panel part. Fig.1 (b) shows the electric circuit of detection part, and Fig.1 (c) is that of touch panel part.

Table 1 compares the characteristics of proposed method with conventional method. From this table, it is understood that the number of parts and the number of connection lines are reduced compared to conventional model. The connection lines are reduced to four from five. In general, 10 or more transistors are used for design of an operational amplifier ${ }^{(6)}$. Assuming 10 transistors are used for the design of an operational amplifiers, 42 electric parts are used to design touch panel circuits in conventional model. In contrast, only 17 parts are used in this model. Next, the details of the circuits of proposed model will be mentioned by the use of Figs. 1 and 2 .

As shown in Fig.1 (a), line number for connecting between panels is four. One line is reduced compared with conventional model. This is only one line reduction, but it will be useful. This is because that it corresponds to the cost reduction if many panels are used. Fig.1 (b) shows the detection part. About four lines of [100 kHz], [GND], [S1], and [S2], the details are mentioned here. The line [GND] is the ground line of the circuit. The line [100 kHz] outputs $100 \mathrm{kHz}, 12 \mathrm{~V}$ clock. This is generated by the IC 7555 and 4404 as shown in Fig.1 (b). This clock is supplied to every panel parts. The lines [S1] and [S2] flows current from panel parts. The lines are connected to many touch panels. When human touches a panel, the currents of [S1] and [S2] flow. If human touches the different position panel, the currents also change. Thus, by detecting the currents, we can understand which panel is touched. How to determine the panel number will be mentioned next chapter [3. Experiment]. In this study, instead of detecting the currents, the voltage $V_{r 1}$ of resistance $R_{1}$ is detected, and the $V_{r 2}$ is also detected as shown in Fig.1 (b). 
Fig.1 (c) shows the circuit of panel part. This is divided into three parts. They are shown in Figure 2.

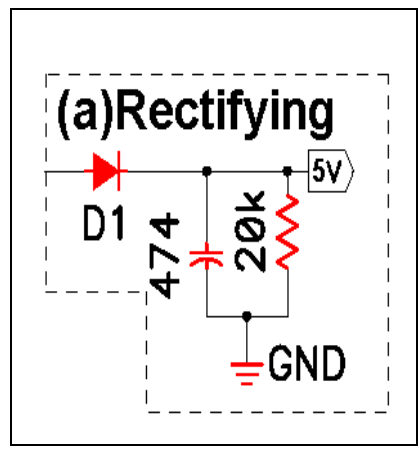

(a) Rectifying part

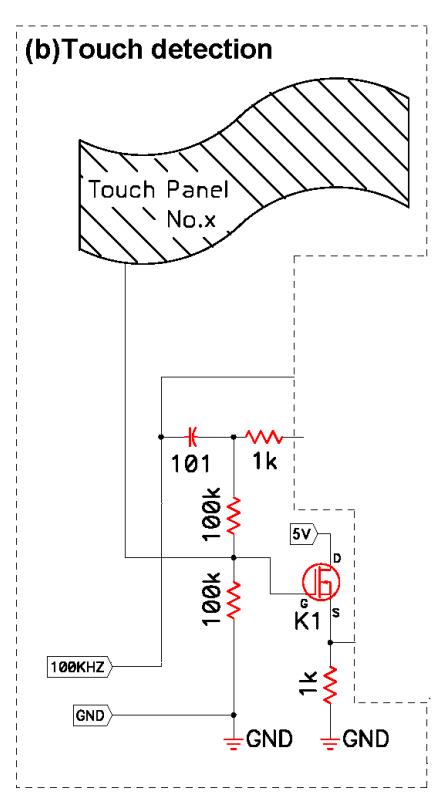

(b) Touch detection part

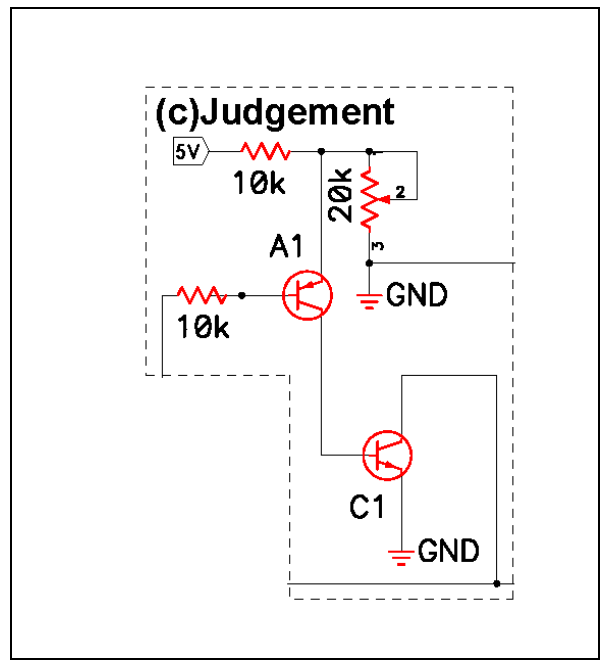

(c) Touch judgement part

Figure 2 Details of touch panel circuits.
Fig.2 (a) displays the rectifying part. In this part, direct current $5 \mathrm{~V}$ is generated from $[100 \mathrm{kHz}]$ clock. As understood from this figure, the circuit is simple. The parts number is small. By the use of this circuit, a DC line is eliminated in this model, although the conventional model needs the DC line ${ }^{(5)}$.

In Fig.2 (b), a current flows from touch panel film to ground though human body, if a human touches a panel. In this circuit, the voltage of source of FET (K1) lowers, if human touches a panel. Only a FET is included in this circuit, and the number of parts is small. Thus, the circuit is simple, and the power consumption is low. The low power consumption is effective according as the panel number increases. Fig.2 (c) displays the judgement part. In this parts, only two transistor are used.

Thus, the number of parts is smaller than that of conventional model as shown in Table 1. The real circuit incorporating the above improvements is designed. The prototype is made for the test of our validity. The picture is shown in Figure 3.

The circuit is designed by discrete electronic parts, so the size is large. However, it is easy to make IC of this circuit, so the size of circuit will be very small and it will be pasted on the surface of touch panel film in future. In this prototype, 20 touch panel films and 20 panel circuits are made. By the use of this, the experiment is practiced as follows.

\section{Experiment}

\subsection{Experiment to identify the positon of the touch} panel

The validity of proposed model is confirmed by the prototype in Figure 3.

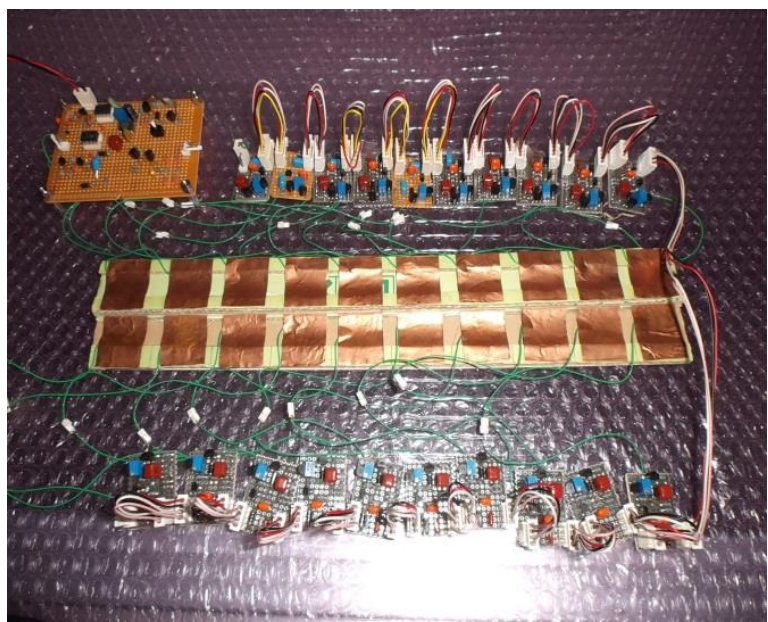

Figure 3 Prototype for testing. 


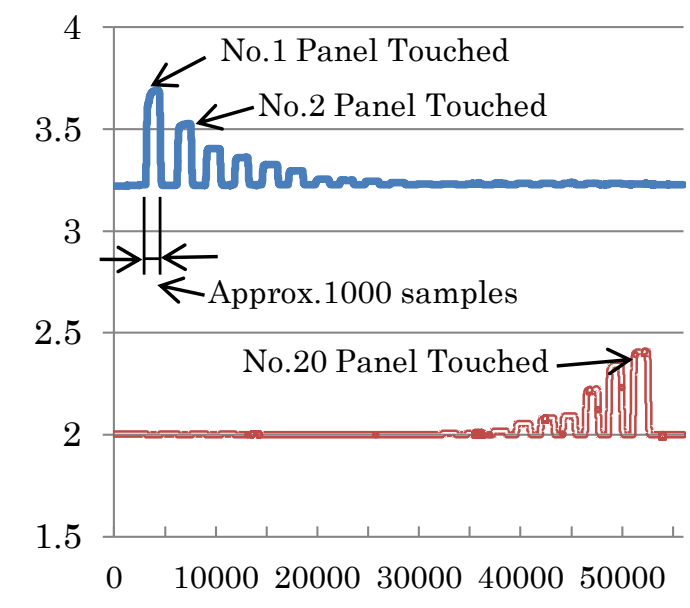

Figure 4 Detected voltage $V_{r 1}$ and $V_{r 2}$.

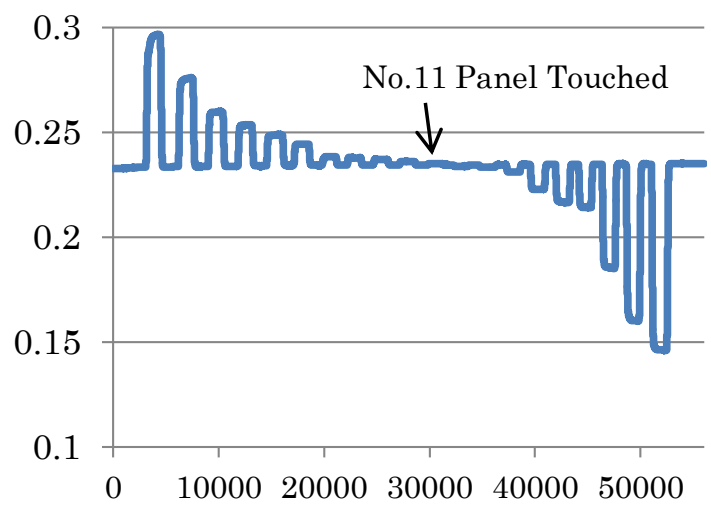

Figure 5 Detected Ratio.

By the connection of data logger to the prototype circuit, the voltage $V_{r 1}$ and $V_{r 2}$ in Fig.1 (b) are measured. Here, the current $i_{1}$ and $i_{2}$ are easily obtained by the equation of $V_{r 1} / R_{1}$ and $V_{r 2} / R_{2}$. The values of voltage $V_{r 1}$ and $V_{r 2}$ are measured at the same time. Figure 4 shows the voltage $V_{r 1}$ and $V_{r 2}$ when 20 sheets of the touch panel have been touched in order. The horizontal axis represents the sampling No., and the sampling period is $1 \mu \mathrm{s}$. In this experiment, the material, whose electrical characteristics is equivalent to human finger, is used instead of real human finger. The material is touched to 20 panels in order from panel No.1 to No.20 at fast interval. The interval is $2.5 \mathrm{~ms}$ (2500 samples). The touched time is $1 \mathrm{~ms} \quad(=1000$ samples).Here, Ratio is defined as follows ${ }^{(4)}$.

$$
\text { Ratio }=\frac{\left(i_{1}-i_{2}\right)}{\left(i_{1}+i_{2}\right)}
$$

From the voltages $V_{r 1}$ and $V_{r 2}$ in Fig.4, the current $i_{1}$ and $i_{2}$ are obtained. By substituting $i_{1}$ and $i_{2}$ into Equation (1), we 8 get Ratio. Each Ratio of 20 panels is shown in Figure 5. Here, let the difference between Ratio of Panel No.x and Ratio of Panel No. $x+1$ be $\Delta$ Ratio $_{x, x+1}$. When $x=11$, the value of $\Delta$ Ratio $_{x, x+1}$ is 0.0070 . The value is the smallest. We define it $\Delta$ Ratio $_{\min }$. In practice, the value of Ratio has a variation even if the same panel is touched. Let the maximum value of the variation be $\varepsilon_{\max }$. In case of ( $\Delta$ Ratio $_{\min }>\varepsilon_{\max }$ ), each panel can be distinguished from the other panels. This will be verified at next chapter $<3.2>$.

\subsection{Verification of maximum panel connection number}

The maximum panel connection number is verified in this chapter. By the use of the law of propagation of errors, the maximum error $\varepsilon_{\max }$ of this method is estimated. Equation (1) is a function of $i_{1}$ and $i_{2}$, so it is represented as Equation (2). When $i_{1}$ and $i_{2}$ include error $\Delta i_{1}$ and $\Delta i_{2}$, the ratio Ratio $o_{\text {er }}$ with error is represented as Equation (3).

$$
\begin{aligned}
& \text { Ratio }=f\left(i_{1}, i_{2}\right) \\
& \text { Ratio }_{\mathrm{err}}=f\left(i_{1}+\Delta i_{1}, i_{2}+\Delta i_{2}\right)
\end{aligned}
$$

By Taylor expansion of Equation (3), Equation (4) is obtained.

$$
\begin{aligned}
f\left(i_{1}+\Delta i_{1}, i_{2}+\Delta i_{2}\right) & =f\left(i_{1}, i_{2}\right) \\
& +\frac{1}{1 !}\left(\frac{\delta}{\delta i_{1}} \cdot \Delta i_{1}+\frac{\delta}{\delta i_{2}} \cdot \Delta i_{2}\right) \cdot f\left(i_{1}, i_{2}\right) \\
& +\frac{1}{2 !}\left(\frac{\delta}{\delta i_{1}} \cdot \Delta i_{1}+\frac{\delta}{\delta i_{2}} \cdot \Delta i_{2}\right)^{2} \cdot f\left(i_{1}, i_{2}\right) \\
& +\cdots
\end{aligned}
$$

From Equation (2), (3), and (4), the error $\varepsilon$ is represented as follows.

$$
\begin{aligned}
\varepsilon & =\text { Ratio }_{\text {err }}-\text { Ratio } \\
& =f\left(i_{1}+\Delta i_{1}, i_{2}+\Delta i_{2}\right)-f\left(i_{1}, i_{2}\right) \\
& =\frac{1}{1 !}\left(\frac{\delta}{\delta i_{1}} \cdot \Delta i_{1}+\frac{\delta}{\delta i_{2}} \cdot \Delta i_{2}\right) \cdot f\left(i_{1}, i_{2}\right) \\
& +\frac{1}{2 !}\left(\frac{\delta}{\delta i_{1}} \cdot \Delta i_{1}+\frac{\delta}{\delta i_{2}} \cdot \Delta i_{2}\right)^{2} \cdot f\left(i_{1}, i_{2}\right) \\
& +\cdots
\end{aligned}
$$

By replacing $f\left(i_{1}, i_{2}\right)$ by Equation (1), and ignoring the higher-order terms, we can get the maximum error $\varepsilon_{\max }$ as follows. 


$$
\varepsilon_{\max }=\left|\frac{2 i_{2}}{\left(i_{1}+i_{2}\right)^{2}} \cdot \Delta i_{1}\right|+\left|\frac{2 i_{1}}{\left(i_{1}+i_{2}\right)^{2}} \cdot \Delta i_{2}\right|
$$

Here, using measured current $i_{1}$ and $i_{2}$, we estimate the maximum error $\varepsilon_{\max }$ of prototype circuit. In our experiment, the currents $i_{1}$ and $i_{2}$ are measured 1000 times, from panel No.1 to No.20 in order. For example, as for panel No.1, the average values $i_{1}$ and $i_{2}$ are 3.66344 and 2.00176, respectively. The deviations $\Delta i_{1}$ and $\Delta i_{2}$ are 0.05237 and 0.00053 , respectively. These values are substituted into Equation (6). As the result, maximum error $\varepsilon_{\max }$ of panel No.1 is obtained as follows.

$$
\begin{gathered}
i_{1}=3.66344, i_{2}=2.00176 \\
\Delta i_{1}=0.05237, \Delta i_{2}=0.00053 \\
\varepsilon_{\max }=\left|\frac{2 \cdot 2.00176}{(3.66344+2.00176)^{2}} \cdot \Delta 0.05237\right| \\
+\left|\frac{2 \cdot 3.66344}{(3.66344+2.00176)^{2}} \cdot \Delta 0.00053\right| \\
=0.006654
\end{gathered}
$$

Table 2 Maximum error $\varepsilon_{\max }$ of Ratio of each panel.

\begin{tabular}{|c|c|c|c|c|}
\hline No.1 & No.2 & No.3 & No.4 & No.5 \\
\hline 0.006654 & 0.002016 & 0.001656 & 0.003403 & 0.005871 \\
\hline No.6 & No.7 & No.8 & No.9 & No10 \\
\hline 0.004293 & 0.001473 & 0.003140 & 0.002271 & 0.001245 \\
\hline No.11 & No.12 & No.13 & No.14 & No.15 \\
\hline 0.000756 & 0.001090 & 0.001899 & 0.004444 & 0.006198 \\
\hline No.16 & No.17 & No.18 & No.19 & No20 \\
\hline 0.003839 & 0.005481 & 0.005388 & 0.005307 & 0.006608 \\
\hline
\end{tabular}

Table 3 Value $\Delta$ Ratio $_{\min }$ vs maximum panel number $N$.

\begin{tabular}{|c|c|c|c|c|}
\hline $\begin{array}{c}\text { Maximum } \\
\text { panel } \\
\text { number } \mathrm{N}\end{array}$ & 100 & 298 & 299 & 500 \\
\hline R $_{\text {atio }}$ min & 0.019608 & 0.006667 & 0.006645 & 0.003984 \\
\hline
\end{tabular}

The above calculation of $\varepsilon_{\max }$ is repeated from panel No.1 to No.20. The results are shown in Table 2.

As understood from the table, $\varepsilon_{\max }$ of panel No.1 is the maximum. The value is 0.006654 . As mentioned before, in case of $\left(\Delta\right.$ Ratio $\left._{\min }>\varepsilon_{\max }\right)$, each panel can be distinguished from the other panels. The value of $\Delta$ Ratio $_{\min }$ changes with maximum connected panel number $N$. Table 3 shows the relationship.

From Table 3 , it is understood that the value of $\Delta$ Ratio $_{\text {min }}$ is 006667 when $N=298$. The maximum error $\varepsilon_{\max }$ is 0.006654 as shown in Table 2. From the fact, if $\mathrm{N}<=298, \Delta$ Ratio $_{\min }>$ $\varepsilon_{\max }$. Thus, the maximum number of connected panel is 298 in this prototype circuit. The panel number is much larger than that of conventional model ${ }^{(4)}$. It is enough in practical use. Thus, the validity of our model is confirmed in this experiment.

\section{Conclusions}

Recently, a touch panel switch with the function of bending and addition was proposed. Since the precision was not enough, the improved model was also proposed. However, it has the following problems. (1) Use of many number of electrical parts, and many number of connection lines. Non-confirmation of the validity of the method in a real circuit.

In order to improve them, a new model has been proposed in this study. As the results, the followings are obtained.

(1) Decrement of the number of electrical parts and the number of connection lines. The proposed new circuit system is simple.

(2) Confirmation of the validity of proposed method in a real circuit.

The maximum panel number is about 300. This is much more than that of conventional method.

In future, we should improve the followings for practical use. (1) Decrement of connection lines further. (2) Avoiding wrong detection in case of 2-point simultaneous touch.

\section{References}

(1) Plauche, Madelaine, Udhayakumar Nallasamy, Joyojeet Pal, Chuck Wooters, and Divya Ramachandran, "Speech 
Recognition for Illiterate Access to Information and Technology," International Conference on Information and Communication Technologies and Development (ICTD2006), Berkeley, 2006.

(2) Sho Yokota, Yasuhiro Ohyama, Hiroshi Hashimoto, Jin-Hua She, "The Electric Wheelchair Controlled by Human Body Motion - Design of the prototype and basic experiment-", Proceedings of the 17th IEEE International Symposium on Robot and Human Interactive Communication RO-MAN2008, ISBN:987-1-42442213-5, pp. 303-308, 2008

(3) Ministry of Internal Affairs and Communications : "As a result of 2013 communication use pulse-taking” Ministry of Internal Affairs and Communications information materials, pp. 9-12, 2014

(4) Seiichi Serikawa, Lifeng Zhang : "Proposal of a touch panel switch with the function of bending and addition", $2^{\text {nd }}$ International Conference on ICICI-BME, pp. 8-13, 2011

(5) Mingyu Fan, Daisuke Tanaka, Akira Yamawaki, Seiichi Serikawa : "Simulation of touch sensor switch control circuit”, The $3^{\text {rd }}$ International Conference on ICIAE2015, pp. 223-226, 2015

(6) Hirokazu Yoshizawa : "The basics of design of the CMOS op-amp circuit" CQsyuppan, pp. 109-110, 2007 